\title{
THE EFFECT OF PLASMA SURFACE INTERACTIONS ON PLT PLASMA PARAMETERS
}

\section{MASTER}

\section{PLASMA PHYSITS LABORATORY}

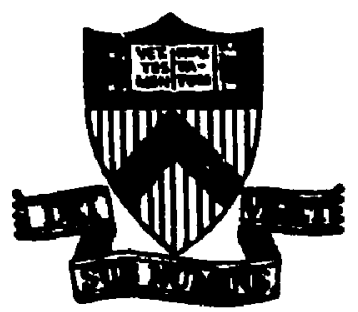

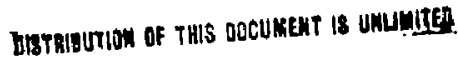

\section{PRINCETON \\ UNIVERSITY PRINCETON, NEW JERSEY}

Thls work wh supported by the U.S. Dewertmens of Energy Contract NO. OE-ACO2-76-CHO 3073. Reproduction, translatlon, publication, use and disposal, ir. whole ar in part. by or for tho United states government is parthitted. 


\section{THE EFFECT OF PLASMA SURFACE INTERACTIONS}

\section{ON PIT PLASMA PARAMETERS}

E. A. Meservey, V. Artinasalam, C. Barnes, K. Bol, N. Bretz,

S. Cohen, P. Colestosk, D. Dimock, H. F, Dylla, D. Eames, P. Efthimion, H. Eubank, R. Goldston, L. Grisham, E. Hinnot, J. Hosea, J . Hovey, H. Hsuan, D. Hwang, F. Jobes, R. Kaita, G. Mecracken*, S. Medley, D. Muelier, N. Sauthoff,

G. Schilling, J. Schivell, I. Stewart**, J. Strachan, W. Stodiek, S. Suckewer, M. Ulrickson, D. Voss, s. von Goeler, G. Zank1*k*

Plasma Physica Laboratory, Princeton University Princeton, New Jersey 08544 USA

W. R. Wampler

Sandia Laboratories

Alouquerque, New Mexico 87115 USA

C. W. Magee

RCA Laboratories

Princeton, New Jersey OB544 USA

\section{ABSTRACT}

In the four years of operation of the PLT tokamak (1976-79), plasma parameters and machine operating conditions have been importantly affected by changes in the choice of limiter material and by the mode of vacuum vessel conditioning. Tungsten, stainless stuel, and graphite liniters have been used. The fractional power lost by radiation and the source distribution of the radiction vary strongly with limiter material, wall * JKAÉr Culinam Laboratory, Abingdon, UR. **Exxon Co., USA.

** On leave: from Max-Planck Institut fur Plasmaphysik, Garching. FPG.

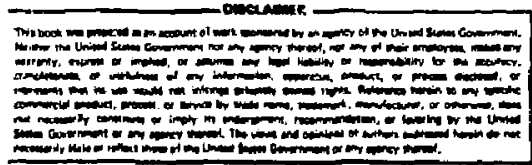


treatment, and gas programming. With tungsten limiters, radiation from partially ionized tingsten atoms can strongly limit the temperature in the center of the plasma; with graphite limiters, radiation is primarily from carbon and oxygen in the outer cold region and not so much from the center, since in the hot core these atoms are totally ionized. With both tungsten and stainiess steel limiters intermediate states exist which have rather unfform radiation source distributions; the characteristics of these distributions are affected by gas programming and by wall conditioning.

Under some conditions the measured energy losses to the limiters and by radiation and neutral particles cannot account for all of the input energy by as much as 30 or 408 .

When the injected gas is changed from $\mathrm{D}_{2}$ to $\mathrm{H}_{2}$, the time constant for disappearance of neutron emission can be slow (35 pulses for a reduction by a factor of three) or fast (4 pulses for a similar factor), depending on previous history of wall treatment.

\section{Introduction}

This paper gives a brief description of the geometry and parameters of the PIT tokamak, reviews some of the last four years' results that are particularly relevant to plasina-boundary interactions, and then concentrates on two specific problems. The emphasis will be not so much on what we know as on what we do not know and where some effort in surface physics might help to provide information. 


\section{PLT Parameters}

Figure 1 shows a schematic view of the cross section of the PLT tokamak vacuum vessel, major radius $130 \mathrm{~cm}$ and minor radius $50 \mathrm{~cm}$. Stainless steel ring limiters are placed inside and outside at $45 \mathrm{~cm}$ radius, and movable limiters at top and bottom, usually placed at $40 \mathrm{~cm}$ radius. These were at first made of tungsten and later of stainless steel or graphite. The plasma is schematically shown as divided into a hot core where energy is well confined and a colder outside ring where energy can be rapidly transported to the limiters and wall either by radiation (primarily from partially ionized oxygen and carbon) or by rapid convection and conduction.

Figure 2 shows typical time dependences of plasma current, 100p voltage, line average electron density, and central electron temperature. Here the current is about $500 \mathrm{kn}$; the voltage decreases gracually to about one volt as the electron temperature increases; the average density is $5 \times 10^{13} \mathrm{per}^{-3}$; and the electron temperature is $1.3 \mathrm{keV}$ in the center of the discharge. The toroidal field for this pulse was 32.5 kilogauss on axis; the pulse length is about 1 second.

\section{Energy Bulance}

In this paper two main problems will be discussed. The first is the important problem of power balance - - where does the energy put into the plasma go, and in what form does it strike the boundary 
materials? Some history is illustrated in Fig. $3[1-5]$. Here we plot on the left side electron temperature and iensity profiles taken with Thomson scattering measurements, and on the right side the radially integrated power radiated from the plasma and the ohmic heating power input lcalculated:from the Te profile, assuming uniform loop voltage and effective $z$ ). The data in Fig. $3(a)$ were obtained using tungsten limiters and represent the classic "radiation-dominated" discharge: hollow electron temperature profile las low as 150 .eV at the center in this casel and essentially a detailed balance between input and radiated power at all radii. The data in Fis. $3(b)$ show a "transport-radiation" case, obtained with graphite liniters top and bottom, where radiation from the hot core region constitutes only a minor fraction ( $\leqslant 208)$ of the input power, the rest moving outward by some sort of anomalous transport into the colder outer shell. Here there are large enough concentrations of partially ionized low-z impurities to radiate most ( $90 \%$ ) of the total energy input. The data in Fig. $3(\mathrm{c})$ were obtained with graphite limiters and pulses in which titanium had been evaporated on parts of the vacuum wall. Here the radiation from the central core region is similar to that of the previous case, but the outer region does not radiate all the energy; so we must find some other mechanism for moving the energy to the material boundaries. It is just here that our ignorance begins to show: not just because we must use "anonalous" (meaning we don't know) to describe the mechanism of transport of a large part of the energy, but we have not yet found where the energy goes. 
Figure 4 illustrates this. We vise an array of thermocouples on the graphite limiters to measure their temperature rise, and we use a scanning bolometer to get a profile of the power emitted in radiation (togather with neutral particle energy). Fisure 4 shows each of these energy losses plotted against the total energy input. For these data the input was divided between ohmic heating and 40-kilovolt neutral beam injection, the spread in Input energies being mostly due to varying amounts of neutral beam power. Here the crosses represent energies deposited on the limiters in individual pulses; the squares are energies depostted on the walls, assuming the intensity at the bolometer to be typical of the whole surface. The circled crosses are the points corresponding to those discharges used for the shot by shot bolometer scan. There is a spread in the data which we cannot explain in detail, but the fraction of energy detected by the bolometers and the limiter thermocouples represents in this run only about 608 of the total input. This means that about 200 or $300 \mathrm{kilojollles}$ of energy (about 408 of the input) have been undetected. Some of the discrepancy can, of course, be ascribed to possible measurement errors, butour best estimate is that a large amount of energy is missing.

There are several speculations on where this energy went; none of them is very believable, but they do point to some Further measurements which should be made: 
(a) Some neutral beam energy is not absorbed by the plasma but is transmitted to the wall at places not sampled by the bolometers. This transmission is not, however, more than about 58 and could therefore account for, at most, $10 \mathrm{kilo-}$ joules. Some beam particles suffer a second charge exchange collision and escape as neutrals before their direction can be changed so as to be measured on the bolometer. These particles carry out $\leqslant 108$ of the beam energy or about another 10 or 15 kilojoules.

(b) Some charged particles that hit the limiters suffer charge exchange collisions on the surface and escape as neutrals with an energy reflection coefficient of perhaps 408 . Because of the geometry, however, about half or more of these particles would re-enter the plasma and therefore would not constitute a net energy loss. This effect might account for 8 or 10 kilojoules.

(c) There may be an increased neutral density near the limiters or at the gas injection ports which would allow a local concentration of charge-exchanged neutrals to carry out an extra amount of energy not reflected in the bolometer measurements. Detailed measurements in these regions have not been made, but it is not likely that they would contribute more than the neutral loss from the rest of the torus. The latter cannot contribute appreciably to the energy loss since some recent velocity-selector measurements by voss [6] indicate a very low neutral loss rate at a location which is typical of the vacuum wall. 
(a) There might be some sort of very fast radial transport across the scrape-off region which allows appreciable numbers of charged particles to reach the wall without first hitting the top or bottorn limiter by moving along the field lines. (Such particles would not reach the bolometer detector through its collimator across the toroidal field.) The loss rate would, however, have to be considerably faster than the Bohm diffusion rate. Measurements on large probes in the scrape-off region by cohen, et al. [7] show an estimated power loss that is only about 18 of the total input.

So the conclusion is that there is in some cases an unexplained sink for about 308 or 40 of the energy.

\section{Eydrogen Retention}

The second specific guestion which will be discussed here is the rate at which one isotope of hydrogen can be replaced by another. This is particularly important in machines where tritium will be used, such as the TFTR and JET tokamaks.

Figure 5 shows some recent neutron production data plotted versus number of pulses. These were ohmic-heating discharges -no neutral beam injection or wave heating. The only gas injected was $\mathrm{H}_{2}$ and this all just before the start of the discharge; so ald the $\mathrm{D}_{2}$ in the discharges comes from the boundary surfaces.

It is clear that, even after 30 or 40 high power shots, significant amounts of deuterium are being evolved from the walls and/or limiters during the flat-top part of the discharge 
(data taken at $200 \mathrm{msec}$ ). Eight days before this run there was a 3-day vaculm opening, then 3 days of pumping, 16 hours of glow discharge cleaning (GDC) with $D_{2}, 13$ hours of low temperature pulse discharge cleaning in $H_{2}$ (TDC), and 8 more hours of GDC in $D_{2}$. There was no titantum gettering during the run; there had been gettering before the vacuum opening, but not after.

About two weeks later, during a similar hydrogen run the neutron intensity started at $2 \times 10^{8}$ and dropped by a factor of three in 4 shots instead of 34 shots. The day before this run the machine was run for 8 hours (about $100 \mathrm{pulses}$ ) at high power in $\mathrm{H}_{2}$ and then $\mathrm{glow}$ discharge cleaned for 8 hours in $\mathrm{H}_{2}$. During the previous two weeks there had been 48 hours of GDC in $D_{2}$ and considerable high power running in $D_{2}$. It appears that glow discharge cleaning is effective in removing deuterium from the wall. Extrapolation to other conditions must be done very cautiously, however, since the effectiveness of the cleaning will depend on the relative impact energy of glow discharge particles and main discharge particles (i.e., discharge conditions) and also on the flux of main discharge particles relative to trapping sites (i.e., the surface composition and condition of the wall). These two different rates of clean-up correspond rather closely to those obtained a couple of years ago by cohen et al. [8-11] measuring $\mathrm{H}_{2}$ and $\mathrm{D}_{2}$ partial gas pressures after the discharge. PLT did not have glow discharge cleaning or titanium gettering at that time. A slow time constant for decrease of $\mathrm{H}_{2}$ in $\mathrm{D}_{2}$ discharges was obtained after 4 months running in $\mathrm{H}_{2}$. A fast time constant was obtained after a long ( 6 week) vacuum opening, followed by 4 days pulse discharge cleaning in $\mathrm{H}_{2}$ and 8 days high energy pulsing in $\mathrm{H}_{2}$. 


\section{Summary}

In summary, two points may be stressed:

There seems to be an undetected component in the energy bialance, and we do not know where or in what form this energy strikes the boundary materials. Until we know this, we can. only speculate about the mechanism for transfer.

Empi:ical data on the rate of hydrogen isotope evolution from the PLT walls show two very different time constants which can be correlated with glow discharge cleaning (but not uniquely since they have not been well correlated with models that explain the differences in terms of machine wall history and discharge conditions).

Resolution of these questions may require some more refined or different surface-plasma measurements.

\section{Acknowledgements}

The continued support of M. B. Gottlieb and H. P. Furth is gratefully acknowledged.

These measurements and analysis would have been completely impossible without the able assistance of the data processing group under Fred seibel and the technical ability of the technician crew under J. M. Perron to keep the PLT tokamak running .

This work was supported by U. S. Department of Energy Contract No. DE-ACO2-76-CHO3073. 


\section{References}

[1] H. Hsuan, et al., in Joint Varenna-Grenoble International Symposium on Heating in Toroidal Plasmas (Centre D'tudes Nucleaires de Grenoble, Grenoble, France, 1978) Vol. II, $87-96$.

(2) R. Bol, et al, in Plasma Physics and Controlled Nuclear Fusion Research (Proceedings Seventh International Conference, Innsbruck, Austria, 1978) Vol. I, IAEA, Vienna, 1979), $11-32$.

[3] V. Arunasalam, et al., in Eighth European Conference on Controlled Fusion and Plasma Physics (Institute of Plasma Physics, Czechoslovakia Academy of Science, Prague, 1978) Vol. II, 17-28.

[4] E. Hinnov, et al., Plasina Physics 20 (1978) 723-734.

i5] H. Eubank, et al., in Plasma Physics and Controlled Nuclear Fusion Research (Proceedings Seventh International Conference, Innsbruak, Austria, 1978) Vol. I, IAEA, Vienna, 1979) $167-197$.

[6] D. E. Voss and S. A. Cohen, 4 th International Conference on Plasma Surface Interactions in Controlled Fusion Devices (Garmisch-Partenkirchen, F.R.G., April 1980) Faper J-1.

[7] S. A. Cohen, et al., 4th International Conference on Plasma Surface Interactions in Controlled Fusion Devices KGarmischPartenkirchen, F.R.G., April 1930) paper A-1.

[8] s. A. Cohen, et al., J. Nucl. Nat. $\underline{76477}$ (1978) 459-471. 
19] V. A. Simonov, B. N. Shvilkin, G. P. Kutukov, Plasma Phygics and Controlled Nuclear Fusion Resear in (Proc. Confi. Salzburg, 1961) Vol. I, IAEA (1961) 141 .

[10] G. M. MeCracken, S. J. Fielding, S. K. Erents, A. Pospieszczyk, P. E Stott, Nucl. Fusion 18 (1978) 35.

[11] E. s. Marmar, J. Nucl. Mat. 76877 (1978) 59-67. 


\section{PLT SCHEMATIC}

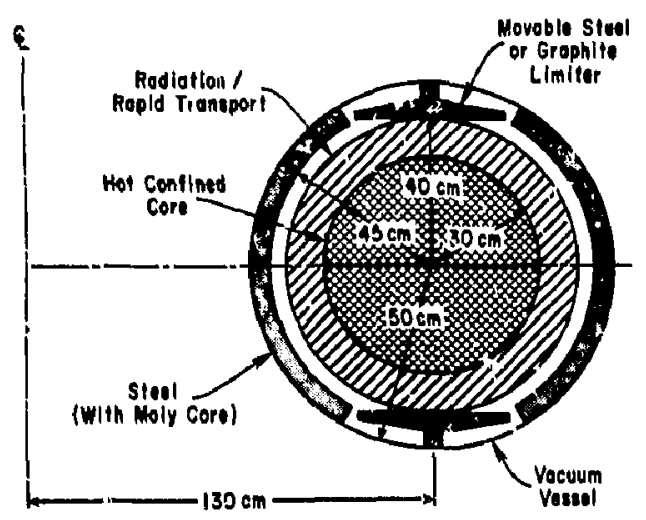

Fig. 1. PLT tokamak cross-section showing the geometry of the Iiniter lucations and a schematic i epresentation of hot and cold plasma regions.

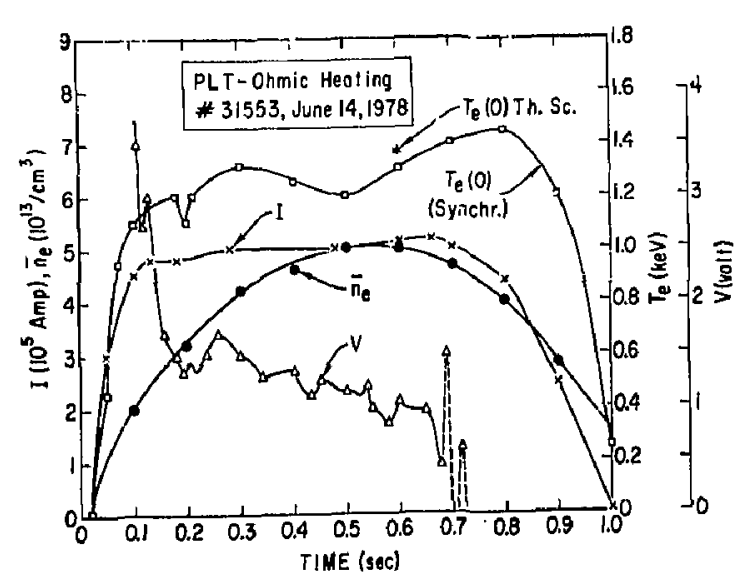

(PPPL-803005)

Fig. 2. Time dependence of various PLT parameters for a typict.1 good discharge: plasma curren: $x, 1$-turn loop voltage $\Delta$, linear average electron density from microwave interferometer •, central electron temperature from synchrotron radiation $\square$, and from Thomson scattering * . 

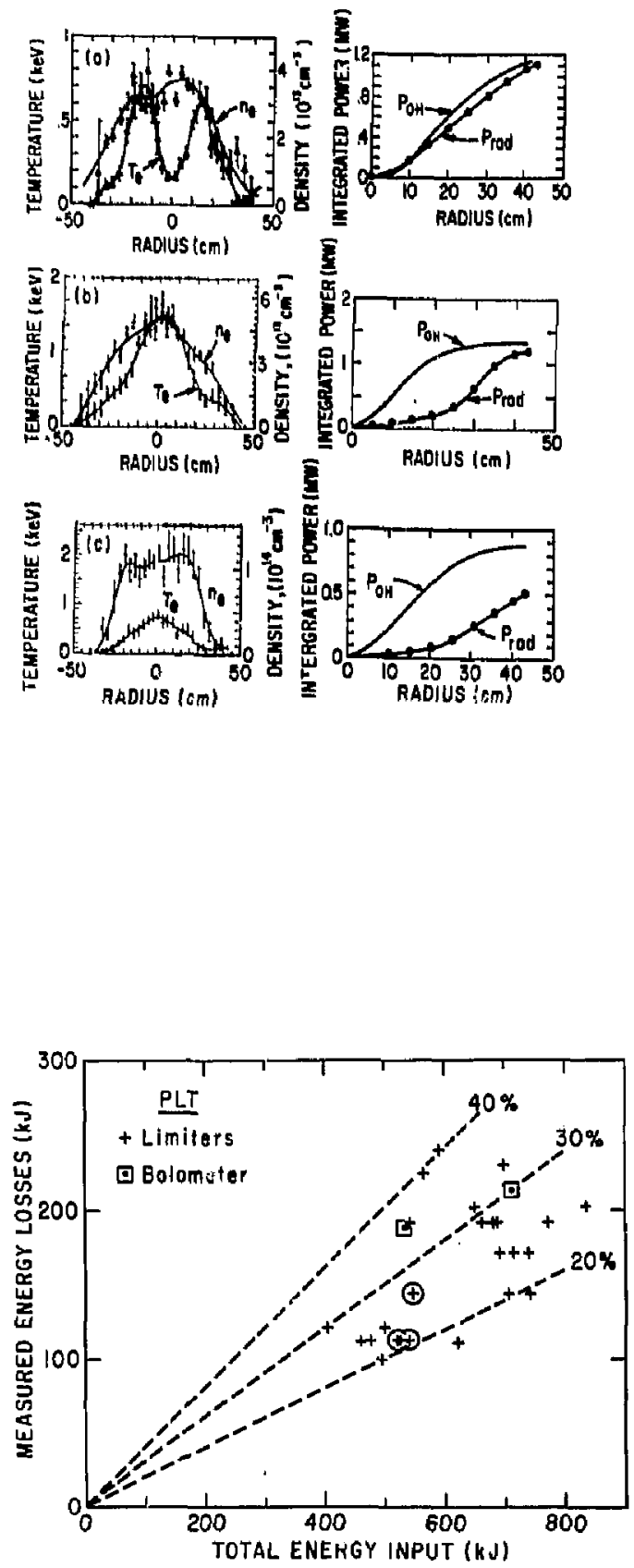

(PPPI-793024)

Fig. 3. Radial profiles of electron temperature and density from Thomson rattering and bolometer measure $\because+s$ of radiated power for ohmlca: y heated discharges for three d. iferent cases:

(a) tungsten limiters showing the hollow tenperature profile and totally radiation-limited discharges;

(b) graphite limiters, peaked temperature profile, large power radiated from outside, ittile radiation from ho' center:

(4) graphite limiters, titanium evaporated on wills between discharges, low-power radjated from center, power radiated from outside distinctiy less than power input.
(PPPL-803601)

Fig. 4. Energy deposited on limiters ( + ) and on wall (scanning bolometer measurements) (m) vs total input energy from ohmic heating and neutral beams. Circled points $(t)$ ire limiter measurements corresponding to the actual pulses on which the boiometer scan was made. Lines snow percentage of input energy. 


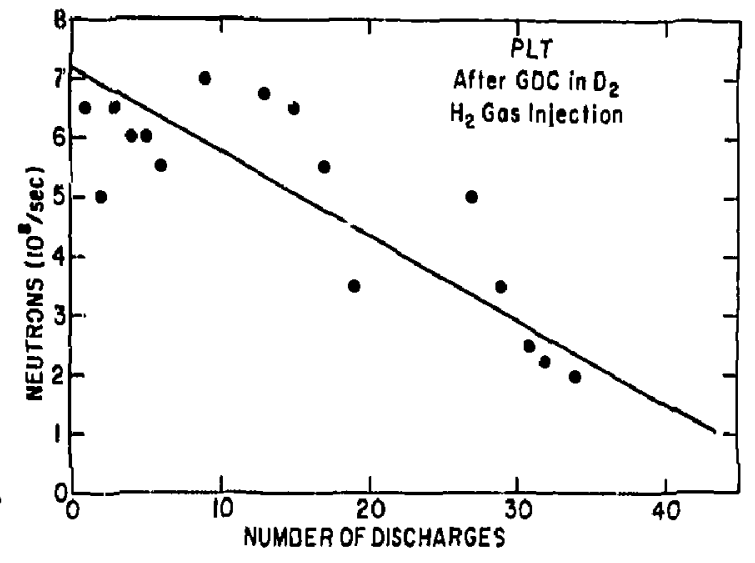

(PPPL-803602)

Fig. 5. Neutron emission after giow discharge cleaning in $\mathrm{D}_{2}$ vs number of discharges, $\mathrm{H}_{2}$ was injected, $\mathrm{D}_{2}$ gas evolved from walls or 1imiters. The initial neutron flux implies almost pure $\mathrm{D}_{2}$ discharges during the steady state (datí taken at 200 msec). 